\title{
Supporting Job Mediator and Job Seeker through an Actionable Dashboard
}

\author{
Sven Charleer ${ }^{*}$ \\ Computer Science Dept., KU Leuven \\ Leuven, Belgium \\ sven.charleer@gmail.com
}

\author{
Francisco Gutiérrez \\ Computer Science Dept., KU Leuven \\ Leuven, Belgium \\ francisco.gutierrez@kuleuven.be
}

\author{
Katrien Verbert \\ Computer Science Dept., KU Leuven \\ Leuven, Belgium \\ katrien.verbert@kuleuven.be
}

\begin{abstract}
Job mediation services can assist job seekers in finding suitable employment through a personalised approach. Consultation or mediation sessions, supported by personal profile data of the job seeker, help job mediators understand personal situation and requests. Prediction and recommendation systems can directly provide job seekers with possible job vacancies. However, incorrect or unrealistic suggestions, and bad interpretations can result in bad decisions or demotivation of the job seeker. This paper explores how an interactive dashboard visualising prediction and recommendation output can help support the dialogue between job mediator and job seeker, by increasing the "explainability" and providing mediators with control over the information that is shown to job seekers.
\end{abstract}

\section{CCS CONCEPTS}

-Human-centered computing $\rightarrow$ Interactive systems and tools; Visualization.

\section{KEYWORDS}

Recommender systems, information dashboards, HCI, explanations

ACM Reference Format:

Sven Charleer, Francisco Gutiérrez, and Katrien Verbert. 2019. Supporting Job Mediator and Job Seeker through an Actionable Dashboard. In Proceedings of ACM IUI 2019 conference (IUI'19). ACM, New York, NY, USA, Article 4, 11 pages. https://doi.org/10.475/123_4

\section{INTRODUCTION}

Due to the abundance of job vacancies online, job recommender systems have become a well researched area [31, 32, 37]. Job seekers can access these recommendations through various job sites and mailing lists, provided by government mediation services and recruitment companies. Meanwhile, recruiters can receive people suggestions for specific job vacancies. These recommender systems are supported by the data provided by the job seekers, explicitly through submitted profiles and CVs, but also implicitly through for instance online activity behaviour [24].

Current recommender systems often behave as a "black box": i.e. recommendations are presented to the users, but the rationale for

*Joint first author.

Permission to make digital or hard copies of part or all of this work for personal or classroom use is granted without fee provided that copies are not made or distributed for profit or commercial advantage and that copies bear this notice and the full citation on the first page. Copyrights for third-party components of this work must be honored.

For all other uses, contact the owner/author(s).

IUI'19, March 2019, Los Angeles, USA

(c) 2019 Copyright held by the owner/author(s)

ACM ISBN 123-4567-24-567/08/06.

https://doi.org/10.475/123_4 selecting recommendations is often not explained to end-users [29]. Several researchers have shown that explanations and user control are needed to support the interpretation of the data and decisionmaking, particularly in high-risk domains that go beyond typical e-commerce recommendations, [23] or in day-to-day user interfaces such as music recommenders that enable end-users to steer the recommendation process with additional input and feedback [22, 25].

In this paper, we present the design and evaluation of an interactive visualisation that can help explain the reasoning behind job recommendations and predictions. The dashboard has been elaborated to be used by on-location recruiters and job mediators to help job seekers better understand the requirements and details of job recommendations. The overall objective is to support the dialogue between "expert" and "laymen" [9]: the expert can become the intermediary between job recommendations and job seekers in order to avoid misinterpretation and incorrect decisions on behalf of the data.

To explore how such a dashboard could benefit job mediation sessions, we collaborated with a regional government job mediation service. Our study follows a user-centred approach, consisting of the following steps:

(1) A preliminary study to understand the workings of the job mediation sessions and first impression of the initial dashboard.

(2) A rapid-prototyping approach to develop and improve the dashboard to better meet job mediator needs.

(3) A study with job mediators to evaluate a set of design goal based in a preliminary study.

This paper is organised as follows: we first present related work in the area of interactive visualisations for recommender systems as well as job recommendation. We then elaborate on the job mediation setting that was used for our study. Section 4 presents the findings of our preliminary study that captures the workings of the mediation service and feedback to a first developed dashboard. Based on our findings, Section 5 sets three design goals to create a new design of the recommender and prediction dashboard: providing control over the message to the job mediator, clarifying the reasoning behind the recommendations and predictions, and supporting the mediators in their workflow. Section 6 reports on the evaluation of the dashboard with 12 job mediators in order to better understand their perceived usefulness and integration with their workflow.

\section{RELATED WORK}

Recommender systems can benefit from interactive data visualisation techniques to support human-recommender interaction [16]. 
Data visualisation can help users to gain new insights [10], and support open-ended explorations and serendipitous discoveries [39]. Providing a rich visual interface can significantly reduce the effort required from users concerning exploratory tasks [40]. Multiuser data visualisation approaches help users collaborate around data [38] for casual exploration [19] or expert use [26]. Collaborative data visualisation can also facilitate and support dialogue between expert and laymen [9]. Moreover, explicit control over the recommended items has shown to result in systems with better acceptance from the users [15], allowing users to have better performance while finding relevant items [41] and granting an increase of engagement and user-experience [29]. In this paper, we elaborate on the explainability [23] of job predictions, for job mediators and job seekers with different educational backgrounds. We also explore how to support the dialogue between job mediators and job seekers through an interactive dashboard visualising job recommender output.

\subsection{Interactive visualisations for recommender systems}

In recent years, several interactive visualisations have been elaborated to support both transparency and user control over recommendations [16]. Particularly in high-risk domains such as health-care and financing, the use of such visualisations has been argued to be very crucial [18]. EpistAid [12] is a prominent example that supports the screening process of physicians by using an interactive visualisation to understand the relevance feedback algorithm. The system improves the answering of clinical questions by promoting user interaction, showing the usefulness of using visualisation to explain recommendations. Several other visualisations focus on improving the accuracy of recommendations with both explanations and support for user control. PeerChooser [28] and SmallWorlds [13] are two approaches that focus on explaining the inner logic of collaborative filtering recommender engines to users. Both systems allow users to interact with representations of relations between items and other users to support transparency and user control. PeerChooser uses a graph-based visualisation to represent these relationships. SmallWorlds allows users to explore the relationships between recommended items and similar friends in multiple layers of similarity.

Also, many visualisations have been developed to interact with hybrid recommender systems. TasteWeights [6] is a system that allows users to control the impact of different algorithms as well as various input data sources on the recommendation results, eliciting preference data and relevance feedback from users at run-time to adopt recommendations. LinkedVis [7] is a prominent example that also investigates the use of interactive visualisations for job recommendations. This idea can be traced back to the work of Schafer et al. [34] on meta-recommendation systems, where users are provided with personalised control over the generation of recommendations by altering the importance of specific factors on a scale from 1 to 5. Similarly, SetFusion [30] is an example of an interface that allows users to fine-tune weights of a hybrid recommender system to represent the relationships between recommendations. MoodPlay [3] is an example of a hybrid music recommender system that integrates different techniques to support explanation and control of affective data. The system allows the exploration of a music collection through latent emotional dimensions, thereby improving acceptance and understanding of recommendations. Yucheng et al. [21] also explored user control in recommendations, creating a visualisation on top of the Spotify recommender system. The system offers different levels of control, where a high-level of control showed to produce better acceptance of music recommendations. In our work, we build on these visual approaches to recommender systems to improve acceptance of recommendations in job mediations settings.

\subsection{Tools to support job recommendation}

Novel approaches for job recommendations include improving the algorithmic side [1, 2, 14, 35] or the filtering for exploration [17], but there is a lack of following a user-centred approach to develop interactive interfaces. To assist the users in their job seeking process, Bakri et al. [4] designed a system with interactive visualisations. The system allows users to filter and get an overview of all posted jobs that met their criteria in less time. Together with data extraction, visualisation becomes a useful component of information retrieval which is key on gaining value and insight. The system designed by Baneres and Conesa [5] is another example related to knowledge and missing skills for a job position. A Sankey diagram is used to visualise the connections between educational programs and needed skills. The system supports users to self-design their long-life learning path according to job and professional expectations. LinkedVis [7] is another very interesting example in this domain, and has been deployed to enable user control over job recommendations. LinkedVis is targeted towards job seekers and has been deployed on top of data from LinkedIn. In our work, we focus on job mediators and use a similar user-centered approach to explore how to support the dialogue between job seekers and job mediators. The overall objective is to provide better control over the presentation and interpretation of recommendations by job mediators.

\section{CONTEXT}

This paper is the result of a collaboration with a regional government job mediation service. This service employs job mediators all over the country to support job seekers. Job mediators are often specialised in specific job sectors. Hence, job seekers are sent to specific offices depending on their background and needs. Job seekers come either voluntarily or by invitation to the job mediation office for a mediation session. These sessions can last from fifteen minutes to two hours, depending on the type of visit. Some sessions involve helping job seekers register with the service for the first time or updating personal information that are typically short. Other sessions are focused on advising job seekers who struggle to find a job.

Some invitations are mandatory, as certain situations will cause job seekers to lose their well-fare unless they are quickly re-employed. The mediation service wishes to provide better support for their job mediators through a new recommendation system. Based on three years of data on the paths of 700000 job seekers (i.e. profile data and type and duration of job acceptance), the mediation service has built a system that predicts a job seeker's chance on finding 
a specific type of job within 120 days. The recommendations are based on data such as age, job experience, educations, and language levels.

The mediation service created a preliminary dashboard for job mediators, depicted in Figure 1. This dashboard visualises the chance of success of finding a specific job within 120 days (Figure 1A). The dashboard provides an overview of the parameters that impact this chance, such as age, region in which the job seeker wishes to work, education, and days unemployed (Figure 1B). Actionable recommendations are listed, as well as the possible impact value the recommendation can have on job chance (Figure 1C). Other job suggestions are hidden, but can be added to the overview (Figure 1D). Figure 2 provides further detail on how these parameters are depicted using a "forest plot" visualisation. A data point matching the value of the job seeker's profile value (e.g. number of days unemployed) is mapped onto the historical data of that parameter. Its Y-value (impact on job chance) is then mapped onto the forest plot, together with the impact distribution of the data.

Our goal is to design a dashboard that attempts to increase trust and acceptance by providing control over, and justification of, the generated predictions and suggestions, while also creating a supportive, collaborative visualisation tool that mediators can use with job seekers during mediation sessions.

\section{PRELIMINARY STUDY}

To get a better understanding of the mediation session and the mediator perception of the first dashboard, we conducted a preliminary study consisting of the following:

- A Customer Journey [27] approach during a one day workshop to get further insights into a typical job seeker-mediator session with five mediators $\left(\mathrm{N}_{\text {mediator }}=5\right)$.

- Observation of hands-on time with the original dashboard (see Figure 1) $\left(\mathrm{N}_{\text {mediator }}=22\right.$, one hour).

- Observations of individual mediation sessions between mediators and job seekers ( $\mathrm{N}_{\text {mediator }}=3, \mathrm{~N}_{\text {customers }}=6,15-30 \mathrm{~min}-$ utes per session).

- A 5-Likert scale (1-completely disagree, 5-completely agree) questionnaire regarding perception of the dashboard and prediction model $\left(\mathrm{N}_{\text {mediator }}=15\right)$ with the questions presented in Table 1.

In the following paragraphs, we summarise the findings grouped by the objectives for interactive visualisations of recommender systems defined by $\mathrm{He}$ et al. [16].

\subsection{Contextual Information}

Mediators report customer profiles are not always up to date, nor do they contain all required information. Therefore, mediators usually do not prepare mediation sessions and take an interview approach to understand customer needs and limitations. This helps get further insights into, for instance, family situations, social behaviour, and appearance. The incompleteness of profiles affects the quality of recommendations. Yet, even complete profiles might generate recommendations that mismatch with the reality that surfaces during these mediation sessions, e.g. due to daycare schedule, attitude, or physical limitations.
Table 1: Questionnaire regarding perception of the dashboard and prediction model

\begin{tabular}{|c|c|}
\hline q1 & $\begin{array}{l}\text { I want to know which factors job seekers can change to im- } \\
\text { prove their chances (e.g. learning a language, training,...) }\end{array}$ \\
\hline q2 & $\begin{array}{l}\text { The job seeker should know which factors they can change to } \\
\text { improve their chances (e.g. learning a language, training,...) }\end{array}$ \\
\hline q3 & $\begin{array}{l}\text { I want to know the factors that impact job seekers' chances } \\
\text { which they cannot control (e.g. age) }\end{array}$ \\
\hline $\mathrm{q} 4$ & $\begin{array}{l}\text { The job seeker should know the factors that impact their } \\
\text { chances which they cannot control (e.g. age) }\end{array}$ \\
\hline q5 & $\begin{array}{l}\text { It is important to understand the reason behind the predictions } \\
\text { based on the profile }\end{array}$ \\
\hline q6 & $\begin{array}{l}\text { It is important for the job seeker to understand the reason } \\
\text { behind the predictions based on the profile }\end{array}$ \\
\hline q7 & $\begin{array}{l}\text { It is important to understand the reason behind the predictions } \\
\text { based on the click behaviour }\end{array}$ \\
\hline q8 & $\begin{array}{l}\text { It is important for the job seeker to understand the reason } \\
\text { behind the predictions based on the click behaviour }\end{array}$ \\
\hline q9 & $\begin{array}{l}\text { It is important to understand the reason behind the predictions } \\
\text { based on the desired job }\end{array}$ \\
\hline q10 & $\begin{array}{l}\text { It is important for the job seeker to understand the reason } \\
\text { behind the predictions based on the desired job }\end{array}$ \\
\hline q11 & I trust the predictions based on the profile \\
\hline q12 & I trust the predictions based on the click behaviour \\
\hline q13 & I trust the predictions for the desired job \\
\hline q14 & $\begin{array}{l}\text { I would like to use the dashboard during sessions with job } \\
\text { seekers }\end{array}$ \\
\hline
\end{tabular}

Participants were moderately positive about using the dashboard during sessions with customers ( $\mathrm{q} 14, \mathrm{M}=3.47, \mathrm{SD}=0.92$ ). It could play a supportive role for mediators by providing a discussion starting point. The suggested actions such as learning a language, broadening regions in which to look for work, or taking a course could help point out how the job seekers can improve their chances.

Support: certain context cannot easily be captured making correct predictions difficult and newly acquired information can result in unrealistic recommendations. The recommendation system can, however, play a supportive role during a mediation session.

\subsection{Transparency}

Mediators have trust issues regarding the "black box" nature of the predictions: trust related to predictions based on the user profile ( $\mathrm{q} 11, \mathrm{M}=2.67, \mathrm{SD}=0.74$ ), click behavior ( $\mathrm{q} 12, \mathrm{M}=2.86, \mathrm{SD}=0.74$ ), and predictions based on the attribute desired job (q13, $M=2.94$, $\mathrm{SD}=0.70$ ) were scored low. Mediators reported to receive occasional odd prediction due to incomplete profiles. "Gut feeling" and mediator experience play an important. Therefore, mediators find it important to understand the reason behind the predictions (q5, $\mathrm{M}=4.34, \mathrm{SD}=.47$ ), which can help explain conflicts or support with their own thoughts. The predictions are based on parameter types that are either actionable (e.g. education, location, and skills) and uncontrollable (e.g. age). These parameters can provide further 


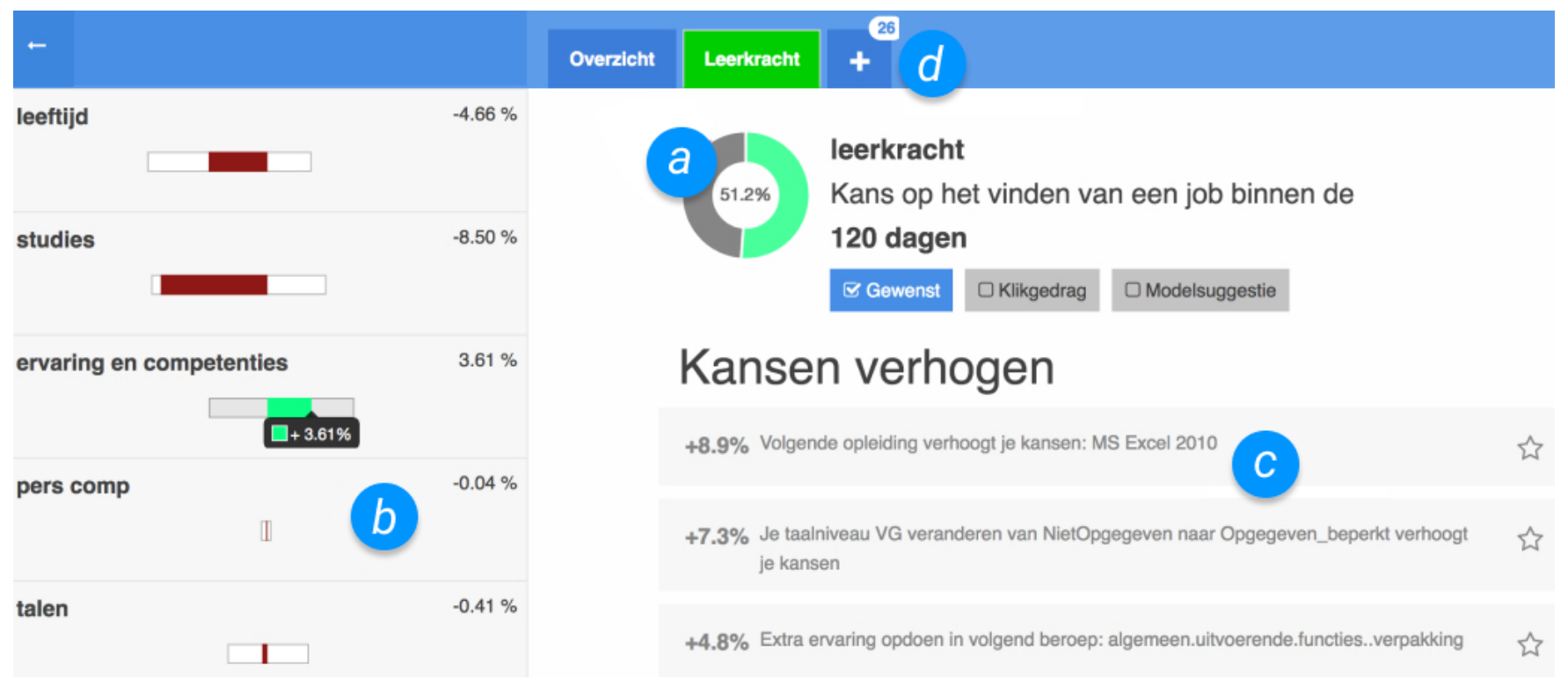

Figure 1: Original (first iteration) dashboard for mediators. A: prediction of chance of success finding this job within 120 days, B: list of parameters that impact chance of success (e.g. age, regions job seeker wishes to work in, education), C: recommendations that can help improve chances (e.g. learn French, add more regions), D: list of more job recommendations which can be added as a new tab

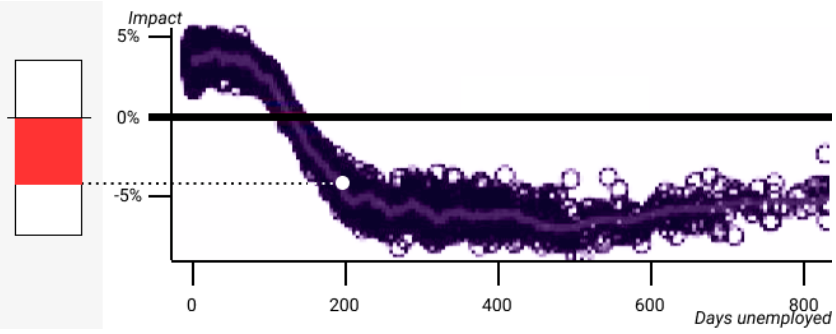

Figure 2: Example of how forest plots are generated: mapping data of "Days unemployed" versus impact percentage on job search prediction. The job seeker is 200 days unemployed, resulting in a negative impact.

insights into why a specific job chance is predicted. Having insights into the actionable parameters was deemed important (q1, $\mathrm{M}=4.46, \mathrm{SD}=0.52)$. Mediators felt uncontrollable parameters were less interesting ( $\mathrm{q} 3, \mathrm{M}=3.4, \mathrm{SD}=1.06)$.

Increasing trust: trust is an important factor. By providing further information through visualising parameter information, job mediators can assess the quality of the recommendation.

\subsection{Controllability}

Mediators are concerned with the impact on job seeker motivation, which is often already very low on arrival at mediation sessions. Showing the dashboard during mediation sessions to job seekers in its current state was not recommended, as job seekers should not see incorrect predictions and unrealistic suggestions. But also

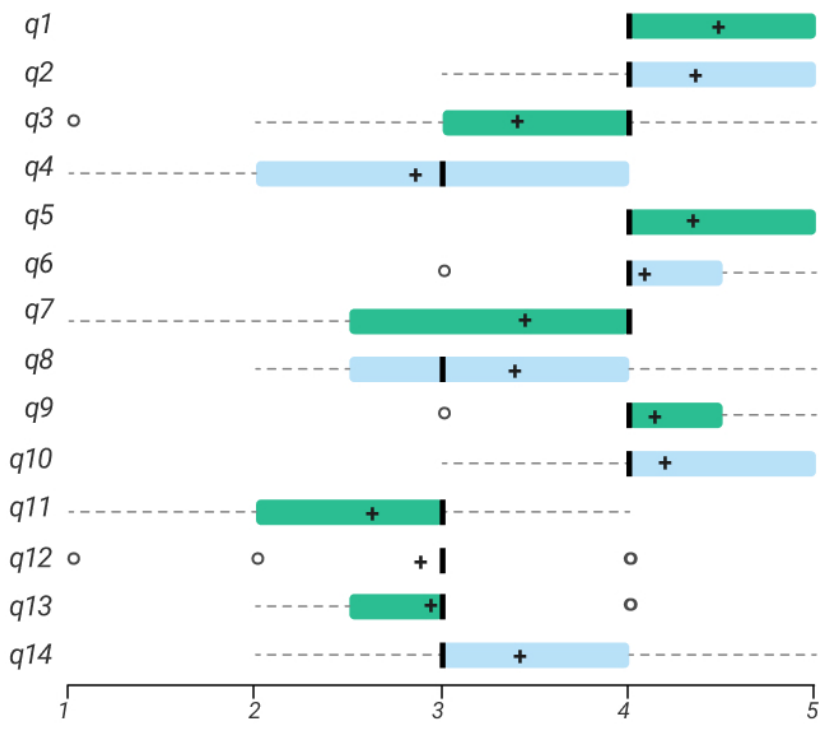

Figure 3: Results from questionnaire regarding perceptions of the dashboard and prediction model $\left(\mathrm{N}_{\text {mediator }}=15\right)$. The dark line indicates the median, “+” the mean, and circles the outliers.

valid information on low job chance, or negative parameters out of job seekers' control (e.g. age) could negatively impact motivation. Providing them with direct access (e.g. through a public on-line service) was deemed unacceptable: highly motivated job seekers 
might overestimate their chances, while demotivated people might have difficulty interpreting data positively.

Control: the mediator needs to remain in control as a filter between the recommender output and the job seeker, to ensure the correct and appropriate data is conveyed without impacting motivation.

\subsection{Justification}

Job seekers sometimes require training and new skills to achieve certain job wishes. Mediators reported that these wishes are sometimes unrealistic. In order to find a job, job seekers must understand their limitation. However, often a mediator's "word" is not enough to convince them. Mediators agreed a visual approach could help convince job seekers, providing a "reality check" to push the job seekers into the right direction. Showing the job seeker the reasons behind a certain prediction was deemed important ( $\mathrm{q} 6, \mathrm{M}=4.14$, $\mathrm{SD}=0.64$ ). However, due to the broad range of educational backgrounds (higher education to none), the way of visualising this information must be carefully considered.

Visual support: visualising detailed information of "behind-thescene" workings of the recommender system cannot only improve trust, but also provide visual support for mediators to convey their message. However, we have to make sure controllability remains with the job mediator to assess the situation and choose what is appropriate to show. The range of visual literacy among job seekers plays an important factor.

\subsection{Diversity}

In its current state, the dashboard provides a list of parameters that influence the prediction (Figure 1B) and a list of actionable recommendations (Figure 1C). As an example, the Languages parameter might indicate there is room for improvement, which will affect the chance of finding a job as Support Engineer. However, mediators required time to find actionable language recommendations within the recommendations list.

Grouping recommendations: similar to $\mathrm{Hu} \& \mathrm{Pu}$ [20], grouping recommendations by parameter type and controllability can help the job mediator find appropriate actions for the job seeker to take.

\section{DESIGN}

Based on the preliminary study, we define following design goals:

- (DG1) Control the message: the mediator filters the information flow to accommodate the situation (for instance to avoid potential demotivation).

- (DG2) Clarify the recommendations: the mediator requires further details into the workings of the recommendations and predictions.

- (DG3) Support the mediator: the dashboard assists the mediator during the mediation session, providing visualisations that can support his/her message.

The dashboard design (see Figure 4) follows the visual informationseeking mantra "Overview first, zoom and filter, then details-ondemand" [36]. First, a list of job recommendations is shown at the top of the dashboard. The user-selected job is indicated by a thick blue line (in this example, "Sales"). For this selected job recommendation, following $\mathrm{Hu}$ and $\mathrm{Pu}$ [20], two groups of parameters are visualised: actionable and non-actionable.
Actionable parameters indicate parameters of which the job seeker has control over, e.g. the region in which he/she wants to work, languages that he/she knows, certificates attained. Nonactionable parameters are uncontrollable, such as the job seeker's age.

The actionable recommendations are grouped per actionable parameter. These recommendations help understand the actions that can be taken and the percentage of impact this action could have on job chance. A job seeker could "learn a language", broaden the region in which they search for a job, or take a specific course.

Job and job chance: to address the potential negative impact of the job chance percentage number, a visual representation without numbers is used: blue indicates the current chance of becoming employed in this job, while grey indicates the potential maximum chance the job seeker has, in case he/she fulfils all suggested recommended actions (see Figure 4A). Example: the job seeker's success chance is high (blue) for Cleaning Help, with little improvement left (grey). While the job seeker's chance for Call-center is currently extremely low, the dashboard indicates high success is possible.

Parameters: the parameters are currently visualised through forest plots, visualising the position on the impact axis and the population distribution (see Figure 2 and 5F), but providing no information regarding the impact if the job seeker's "value" changes. Example: In Figure 2, the job seeker is unemployed for 200 days. The negative impact on job chance is about four percent. The forest plot provides no information regarding the impact on job chance when the job searcher remains longer unemployment, nor can the forest plot tell when the tipping point of positive to negative impact on job chance occurred (from the graph we know this happens around 150 days).

To further investigate transparency of predictions, as well as the impact on motivation, we designed two other representations presented in Figure 5C/B.

Parameter version $\mathrm{C}$ (ircle): this parameter visualisation (see Figure $5 \mathrm{C}$ ) splits the parameter population data across the value axis into segments, providing detailed information lost in the forest plot. The simplicity of the visualisation, by indicating positive/negative impact through binary colours, should also be understandable by a wider audience. Example: In Figure 4, the non-actionable "Age" parameter indicates that the young age (19-25, red) of the job seeker is having a negative impact on job chance. However, this representation provides insight into the tipping point: at the age of $26+$ (green) chances will go up.

Parameter version B(archart): This parameter visualisation (see Figure 5B) provides the same information as version $\mathrm{C}$, but adds an extra dimension. While colour indicates the type of impact, the height indicates the amount of impact the parameter has on the total job chance. Example: In Figure 5B, again "Age" as impact on job chance is presented. The added dimension shows that the age range of 26-30 has highest positive impact, while the impact is at its worst in the range of 56-65.

Recommendations: The recommendations (see Figure 4B) are grouped by their relevant parameters. Uncontrollable parameters, such as age and days unemployed, contain no recommendations (see Figure 4C). Controllable parameters do contain a number of recommendations that can be added to the prediction model. Example: In Figure 4B, the "Languages" group contains both the impact of languages known and the suggested actions that could improve 


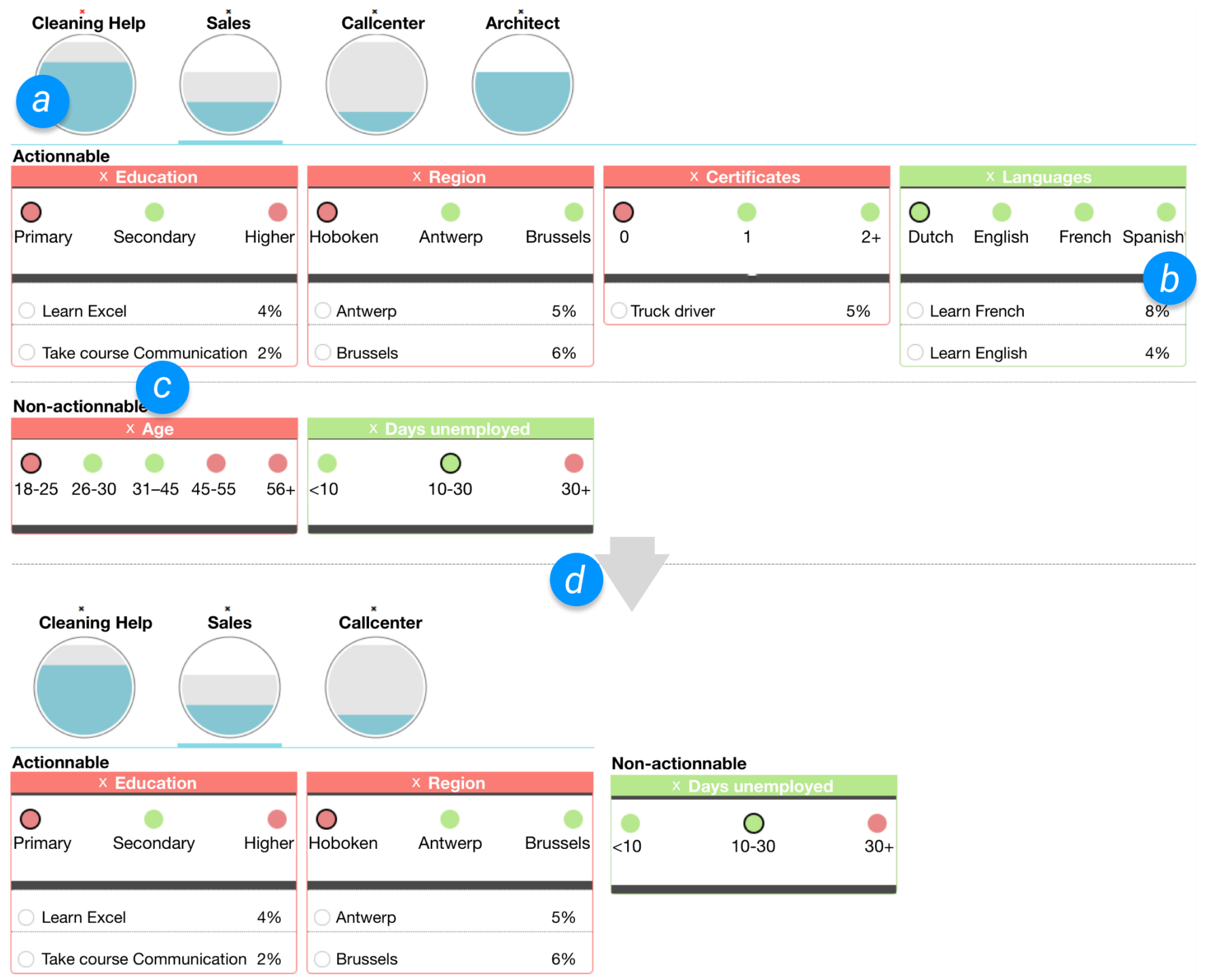

Figure 4: The dashboard suggests four jobs. The bottom image shows the resulting dashboard after manual filtering by the mediator, removing the incorrect prediction (architect), and narrowing down relevant information (according to this mediator) to help the conversation.

chances further. "Learn English" would for instance improve the job seeker's job chance by eight percent.)

Customisation: due to the importance of having full control over the message presented to the job seeker, job mediators can remove parameters and job suggestions using the $x$ next to each item, customising the dashboard to their needs. Example: Figure 4D) is the result of a mediator removing both jobs and parameters from the dashboard, in preparation of a mediation session. The unrealistic job "Architect" and incorrect recommendation "Truck driver" have been removed. The non-actionable parameter "Age" has been removed to avoid demotivation, while "Days unemployed" was left as eye-opener.

\section{EVALUATION}

\subsection{Study Design}

We ran a qualitative evaluation with expert users $\left(\mathrm{N}_{\text {mediator }}=12,10 \mathrm{~F}\right.$, age: $\mathrm{M}=40.7, \mathrm{SD}=9.4$, years experience as mediator: $\mathrm{M}=9, \mathrm{SD}=4.3$ ). Six mediators dealt solely with higher education job seekers (P2, P3, P4, P5, P6, P11), four with secondary to higher education job seekers (P1, P7, P9, P10), and two with job seekers that have no to technical/professional education (P8, P12). Mediators participated in the evaluation sessions individually. Each session lasted half an hour to an hour. The sample size of our study is limited because of constraints of availability of expert users, but inline with the most common sample size of Human-Computer Interaction studies [8]. 


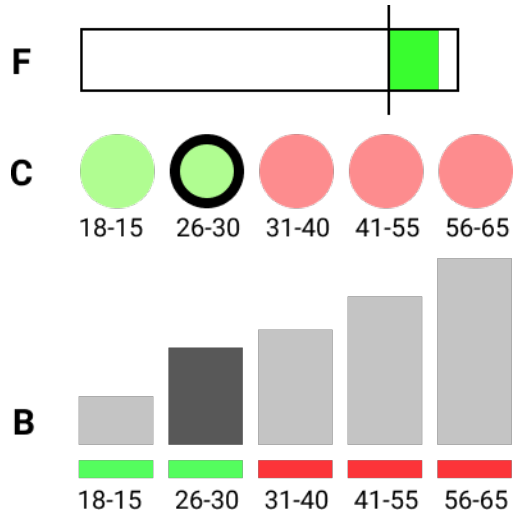

Figure 5: Three ways of presenting the parameter data, with age as example. Forest plot $(F)$ indicates the job seeker's age has a positive impact. Circles (C) segments the data across the age value, indicating positive and negative impact through colours: the job seeker's age is within the positive 26-30 range. Barcharts $(B)$ add the size of impact to each segment through height: the $26-30$ age range has more positive impact than the $18-25$ range.

Table 2: Questionnaire regarding interpretation of visualisations

\begin{tabular}{|c|c|c|}
\hline & question & variable \\
\hline $\mathrm{q} 1$ & $\begin{array}{l}\text { Is the visualisation clear to you (without prior } \\
\text { explanation)? }\end{array}$ & clarity \\
\hline q2 & $\begin{array}{l}\text { Is the visualisation clear to you (after expla- } \\
\text { nation)? }\end{array}$ & $\begin{array}{l}\text { clarity + explana- } \\
\text { tion }\end{array}$ \\
\hline q3 & $\begin{array}{l}\text { Would the visualisation be clear to a job } \\
\text { seeker (without prior explanation)? }\end{array}$ & clarity job seeker \\
\hline q4 & $\begin{array}{l}\text { Would the visualisation be clear to a job } \\
\text { seeker (after explanation)? }\end{array}$ & $\begin{array}{l}\text { clarity job seeker } \\
+ \text { explanation }\end{array}$ \\
\hline q5 & $\begin{array}{l}\text { Would the visualisation be an added value to } \\
\text { the mediation session? }\end{array}$ & added value \\
\hline q6 & $\begin{array}{l}\text { Would the visualisation help motivate the } \\
\text { job seeker? }\end{array}$ & motivates \\
\hline q7 & $\begin{array}{l}\text { Would the visualisation demotivate the job } \\
\text { seeker? }\end{array}$ & demotivates \\
\hline
\end{tabular}

We took a semi-structured interview approach during each session, which consisted out of two parts: 1) feedback on the three parameter visualisations (see Figure 5), and 2) interaction with, and feedback on, the working prototype of the dashboard.

Feedback on parameter visualisations: Through a counterbalanced, within-subject study, participants were shown three examples of each parameter representation (circles chart $=C$, barchart $=\mathrm{B}$, forest plot $=\mathrm{F}$ ) individually. We asked participants to interpret each visualisation out loud and fill out a questionnaire on a 5-Likert scale (1-Completely disagree,5-Completely agree), see Table 2.

The results per visualisation are presented as boxplots in Figure 6. Eleven out of twelve participants were already familiar with the forest plot through the preliminary study. A semi-structured interview approach was used to get further clarifications with each answer.

Interaction with dashboard: participants were also given time to familiarise with the dashboard as shown in Figure 4 and were given extra explanation if necessary. The think-aloud protocol was used while the participant followed a simple scenario: prepare the dashboard for a customer that is recently unemployed, experienced in cleaning, and wishes to change careers if possible. The participants were observed and the activities on screen were recorded. Four jobs were suggested, including an incorrect prediction. A subset of both actionable and non-actionable parameters were visualised. One parameter contained an incorrect suggestion.

\subsection{Control the message (DG1)}

Observation of the participants showed that customisation of the parameters is highly dependent on the mediator: five participants used the negative parameters to support their message, either to push the customer into another direction ("Call-center will be difficult, this parameter will help explain that" (P1)) or to show potential over long term ("She is too young now, but her age will be an advantage for this job" (P3)). Two participants immediately removed these non-actionable negative parameters to avoid demotivation. One participant hid a large number of parameters to focus on the positive, but to also simplify the view for the customer. The semi-structured interview revealed two themes emerged regarding controlling the message: customisation and the importance of the human factor.

6.2.1 Customisation: Mediators stressed the importance of customisation, commenting that "incorrect predictions must be removed" (P2) but also that "unrealistic predictions must go" (P10) as the system can suggest jobs that require steps which are not achievable within a realistic time-frame (e.g. learning a new language might take too long depending on the urgency of finding a job). Such recommendations can also be demotivating. But so can uncontrollable parameters: "Age can be demotivating" (P10)) and "the job seeker might keep staring at the $50+$ years indicated in red" (P7).

Too much information might be difficult to process by certain job seekers (P2). Customisation would help create a simpler and better overview (P2, P3, P9, P12), but one mediator "would like to keep seeing an overview of everything”, while showing a subset of the data to the job seeker (P3). The dashboard might still be too difficult to understand depending on educational background or sector, e.g. "It really depends on the job seeker, I would not show this to someone who is a cleaner, it's too complicated" (P4).

The contextual information gathered during the session can guide the customisation. Depending on the needs and limitations of the job seeker, the dashboard can be altered to support the conversation. "If I know certain jobs do not interest them, I'll remove it" (P7). One mediator (P1) wanted to add a job that is not listed, and see the chance and recommendations in order to use this during the conversation. One mediator would, however, not customise at all: "Let the job seeker react to what they see" (P8), "Why would you want to hide what is relevant" (P8). 


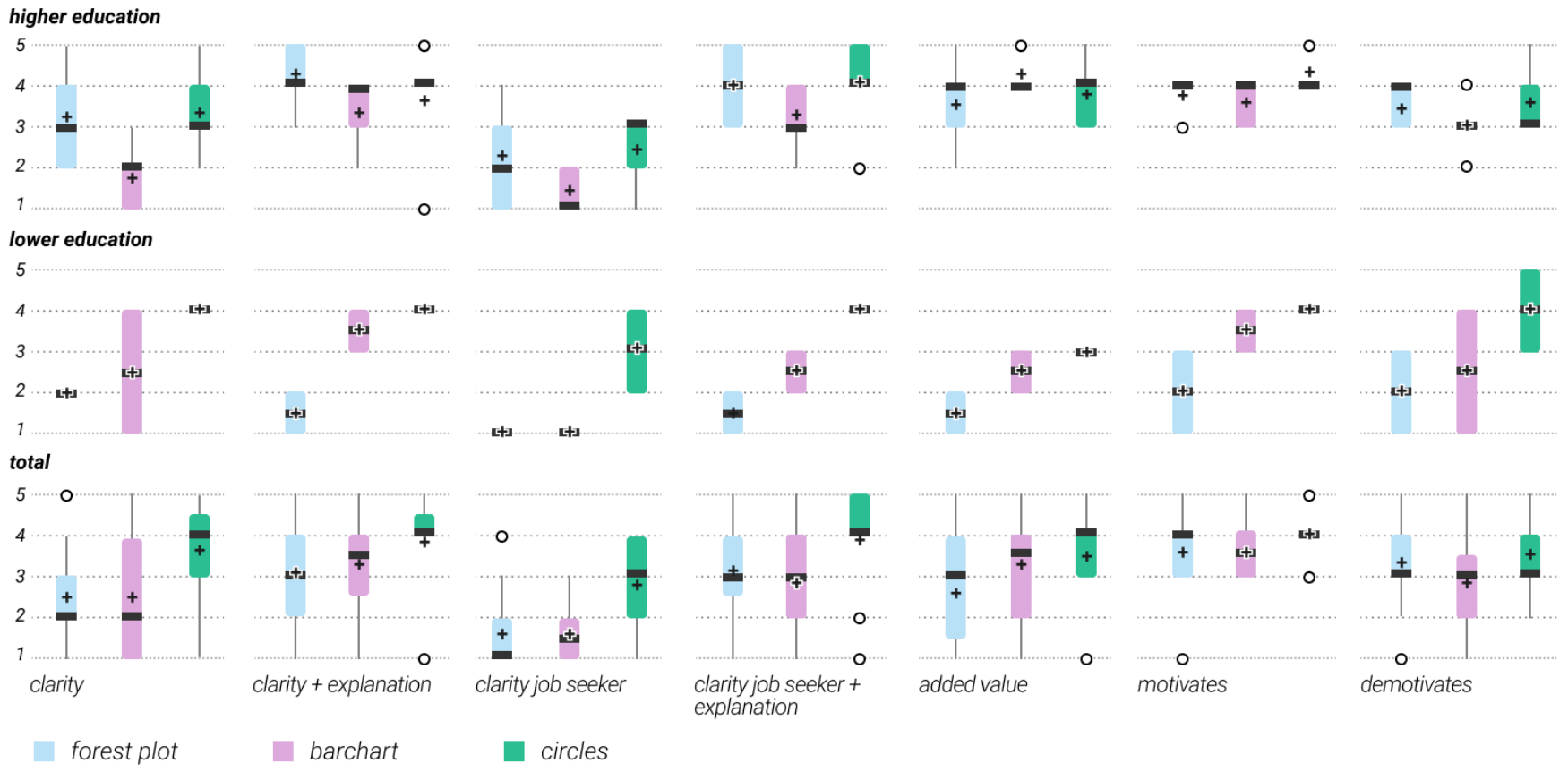

Figure 6: Boxplots of the five-Likert scale questionnaires (1-Completely disagree, 5-Completely Agree) comparing the different parameter visualisations regarding clarity, clarity with additional explanation, clarity towards the job seeker, clarity for the job seeker with additional explanation, added value, positive and negative motivational impact. Top: responses of mediators with job seekers with higher education background. Middle: responses of mediators with job seekers with lower education background. Bottom: the combined results. The dark line indicates the median, “+” the mean, and circles the outliers.

6.2.2 The Human Factor: Mediators want to customise the dashboard. They believe that this human, expert, intervention is required before showing the data to the job seeker. Mediators even go as far as calling it "[...] dangerous if this would replace human estimation of the job seeker's situation" (P1). The dashboard can, however, add to the conversation (P1) but the data in the system "is only part of the information on the job seeker" (P6), and is missing the external context often gathered during the sessions. A job seeker might for instance be "introvert or extrovert" (P6) which can affect their chances and create a mismatch with certain predictions.

Whether or not a job is achievable can be deduced during the session. Job seekers might overestimate the work necessary to fulfill certain recommendations, which the mediators can help assess (P3, P4). But also the difference in job mediators might affect the interpretation: "Some mediators just want people to start working as soon as possible, doing anything" (P4), in which case the needs of the job seeker might (have to) be neglected (e.g. in order to not lose rights to well-fare).

\subsection{Clarify the recommendations (DG2)}

Two themes emerged from the evaluation related to clarification of recommendations and predictions: understanding the visualisation and convincing power.

6.3.1 Understanding the visualisation: The study on parameter visualisations showed that $\mathbf{C}$ (ircles) was considered as the most clear representation to mediators. Mediators mentioned "there is fewer information, which makes it easy to interpret”. Most mediators did not notice the height difference in $\mathbf{B}$ (archarts) at first, but did say it "contains more nuance". "I have more information to support the conversation", but "It is harder to interpret". F(orest plots) scored worse: "I have no idea what actions to suggest to the customer", "It is hard to interpret for which I have no time during a session". Mediators also mentioned there is too much information, such as distribution, which is not useful. Most mediators would not go further than interpret the colours red and green as bad and good.

Mediators were asked to assess how easy it would be for their average type of job seekers to interpret the visualisations. Mediators assumed $\mathbf{C}$ would be somewhat understandable, and were confident they could clarify it. $\mathbf{C}$ was considered useful: "The job seeker will remember this", "There is more nuance to explain what actions to take", "I can use this to convince the customer and show facts". For B, the extra nuance was also considered beneficial, but was considered too difficult for the customer: "There is too much explanation necessary" and "I will lose their focus during the conversation". For F, mediators assumed "It will not stick", but "IT-orientend job seekers might understand" but it would "not [be] usable for job seekers with lower education". While mediators do wish to for job seekers to understand the reasoning behind the predictions, one mediator did mention that "the job seeker does not require to understand every detail". 
Based on the educational backgrounds of their job seekers (see Figure 6), we noticed mediators scored the visualisations better for use with job seekers with higher education ("clarity job seeker + explanation", $M_{C}=4.5, M_{B}=3, M_{F}=4$ ). Mediators with job seekers with lower education scored the visualisations worse ("clarity job seeker + explanation", $M_{C}=4, M_{B}=2.5, M_{F}=1.5$ ), with the biggest difference observed for $F$. However, everyone agreed prior explanation of the visualisations to the job seekers would be necessary ("clarity job seeker", $\left.M_{C}=2.8, M_{B}=1.7, M_{F}=1.7\right)$. Most participants considered all versions to be of added value, with a preference towards $\mathbf{C}$, due to its simplicity. Regarding motivational impact, no clear difference was observed. Participants mentioned "Sometimes the job seeker must face reality", "It can be used as an eye opener", "Showing possible paths can motivate", and "It can reinforce confidence". Participants do believe in some cases the dashboard should not be shown: "If all parameters are negative, this might be too confronting". Finally, we inquired about general preference: participants rated $\mathbf{C}$ highest. $\mathbf{B}$ was ranked second and $\mathbf{F}$ was ranked last.

6.3.2 Convincing Power. Certain information might be confrontational and demotivating. To avoid demotivation, customisation helps the mediator filter the message. However, visualising and showing some of these data can also help the mediator convince the job seeker (P1, P3, P8, P10). It can help confront job seekers, e.g. "they can say what they want, we have it here on black and white, so they will accept it more easily" (P10).

For job seekers who overestimate themselves, mediators can use the dashboard to push them in other directions. Unrealistic expectations of the job seeker can be taken care of, e.g. "[the job seeker doesn't] want to follow a training, it takes too long. But now I can show [without training] it's impossible" (P8). "A complete negative overview can be used to say it'll be difficult [to achieve what he wants]" (P3). Even a positive overview can be beneficial, confronting the job seeker with the fact that there is no reason why he or she should not have found a job yet (P8).

\subsection{Support the mediator (DG3)}

Participants agreed they want to use the dashboard for their sessions. However, the way of using the dashboard highly depends on the mediator and the situation.

The dashboard would be beneficial during the orientation cases. In these cases, the job seeker does not have a good idea of possible career paths. The mediator "can help orientate" (P1) them. In case they are stuck with a specific job in mind, mediators can use the dashboard to show the job seekers "alternate paths and job sectors" (while hiding unrealistic options) that they might have never considered possible.

The dashboard can also be useful when the mediator is stuck. While mediators are guided by their experience, mediators new to the job can use the dashboard as a starting point: "I think it would be an added value for a starter. It will help figure out the steps to suggest to a job seeker" (P1). But even experienced mediators see the benefits. They "can go through the actions they should take [to achieve what they want]" (P1), and "can see really well and clear what is achievable and what is not" (P9). It could help deal "with problem cases" (P11), e.g. when the customer "does not know what he/she wants and is being resistant" (P1). It can provide a guide during the session, helping with "longer conversations" (P8), providing "clarity for the job seeker" (P9) which is "good for long conversations” (P8).

\section{DISCUSSION}

The job recommender and prediction system provides a job seeker with the percentage of chance to find a specific job within a certain timeframe and suggests possible actions to improve this chance. In this work, we provided more control to the job mediator (DG1), improved its "explainability" (DG2), and supported the session between mediator and job seeker (DG3).

Recommender systems with increased "explainability" increase users' trust and provides them with actionable insights. We designed a dashboard that helps mediators understand the predictions, clarify the reasoning to job seekers, and help them take appropriate actions to improve their job chance success (DG2). However, the sensitive nature of the situation, i.e. the broad spectrum of reasons why they are unemployed and/or unsuccessful in finding a job, makes providing the recommendations and predictions to job seekers "as-is" dangerous. Mediators stress the importance of taking into account job seeker motivation and their possible ability of misinterpretation.

Job recommender and prediction system output might not seem as critical as e.g. hurricane forecast [33]. However, our study shows that the human expert plays a crucial role in interpreting and relaying the recommended output. Similarly, Charleer et al. [9] ran into similar concerns regarding the visualisation of student data: it is important to take into account context which is not available within the data, and personality of the user can affect the way data is interpreted. Wrongly interpreted data can result in incorrect (student career) choices and greatly impact their lives. Similarly, job seekers' decisions can be guided by recommendations and predictions, but they could also be "manipulated" by incorrect or unrealistic predictions [11]. Again, each situation might call for a different approach, and this data should be filtered and interpreted correctly by experts to relay the information to job seekers in the most appropriate way (DG3).

These experts become so-called "gatekeepers" of the data. While the dashboard carries the message, it is the mediator that "moulds" the message for maximum positive job chance success based on job seeker needs and requirements. Mediators can leverage the visual nature of the dashboard which provides weight to their arguments. To this end, the mediator must control what is visualised (DG1) to avoid demotivational impact, or simplify the dashboard to support less visual literate job seekers. The dashboard could also provide customisation of the types of visualisation to benefit a broad spectrum of job seekers. Simple visualisations (e.g. Figure 5C) are more clear to a wider audience, while more detailed versions (e.g. Figure 5B/F) can help provide richer insights.

Customisation is used beyond data filtering: it also helps support the different approaches mediators take. It can support the dialogue as the mediator goes back and forth between showing and hiding relevant information, create confrontational situations (so called "reality checks") by revealing negative factors, and assist in soft motivational approaches by eliminating demotivational parameters from sight. No common way of visualising the data would suit the 
approach of every mediator and the contextual situation from each session.

\section{CONCLUSIONS \& FUTURE WORK}

Job mediators are faced with a broad job seeker audience: different educational backgrounds, social situations, and personalities require the mediator to cater to each individual need and context. The incompleteness of customer profiles and the lack of information regarding external factors to the system make visualising job search predictions and suggestions "as-is" difficult. Our work attempts to address this challenge by designing and evaluating a dashboard for use during mediation sessions. Our design attempts to clarify the recommendations and predictions to both mediators and job seekers, supports the conversation between mediator job seekers, and helps mediators control the message they wish to convey depending on the situation context.

Some limitations must be taken into consideration when interpreting our findings. All evaluations were held with job mediators. While the interaction between job mediators and job seekers were observed, we were only able to interview job mediators regarding the dashboard design and interaction. These mediators do, however, have a good understanding of job seekers through years of experience of interviewing multiple job seekers with different needs and situations every day. We argue that their understanding is good enough for support the reported findings on the dashboard and its potential for mediation sessions. With these results, we now have a dashboard which we can deploy in realistic settings in order to get further insights into its impact on mediation sessions.

We invite researchers to explore this explanatory dashboard for a dialogue approach in other domains. These design guidelines are valid beyond mere job recommendations and predictions. Fields where data and recommendations are delivered to experts can benefit from this work in order to create better tools to help experts pass the information onto less knowledgeable parties, e.g. in health (doctor-patient) and education (teacher/staff-student), whereas fields where laymen have direct access to the data such as online banking, expert intervention supported by such dashboards could help with better decision making.

\section{ACKNOWLEDGMENTS}

We would like to thank all mediators of the Flemish PES (VDAB) for their participation and valuable input. Part of this research was supported by Flemish PES (VDAB) and the KU Leuven Research Council (grant agreement C24/16/017).

\section{REFERENCES}

[1] Shibbir Ahmed, Mahamudul Hasan, Md Nazmul Hoq, and Muhammad Abdullah Adnan. 2016. User interaction analysis to recommend suitable jobs in careeroriented social networking sites. In Data and Software Engineering (ICoDSE), 2016 Int. Conf. on. IEEE, 1-6.

[2] Nikolaos D Almalis, George A Tsihrintzis, and Nikolaos Karagiannis. 2014. A content based approach for recommending personnel for job positions. In Information, Intelligence, Systems and Applications, IISA 2014, The 5th Int. Conf. on. IEEE, 45-49.

[3] Ivana Andjelkovic, Denis Parra, and John O’Donovan. 2016. Moodplay: Interactive Mood-based Music Discovery and Recommendation. In Proceedings of the 2016 Conference on User Modeling Adaptation and Personalization. ACM, 275-279.

[4] M. Bakri, Siti Z. Z. Abidin, and Amal Shargabi. 2017. Incremental Filtering Visualization of JobStreet Malaysia ICT Jobs. In Soft Computing in Data Science,
Azlinah Mohamed, Michael W. Berry, and Bee Wah Yap (Eds.). Springer Singapore, Singapore, 188-196.

[5] David Baneres and Jordi Conesa. 2017. A Life-long Learning Recommender System to Promote Employability. Int. fournal of Emerging Technologies in Learning (ifET) 12, 06 (2017), 77-93.

[6] Svetlin Bostandjiev, John O’Donovan, and Tobias Höllerer. 2012. TasteWeights: a visual interactive hybrid recommender system. In Proc. RecSys'12. ACM, 35-42.

[7] Svetlin Bostandjiev, John O'Donovan, and Tobias Höllerer. 2013. LinkedVis: exploring social and semantic career recommendations. In Proceedings of the 2013 international conference on Intelligent user interfaces. ACM, 107-116.

[8] Kelly Caine. 2016. Local Standards for Sample Size at CHI. In Proceedings of the 2016 CHI Conference on Human Factors in Computing Systems (CHI '16). ACM, New York, NY, USA, 981-992. https://doi.org/10.1145/2858036.2858498

[9] Sven Charleer, Andrew Vande Moere, Joris Klerkx, Katrien Verbert, and Tinne De Laet. 2017. Learning Analytics Dashboards to Support Adviser-Student Dialogue. IEEE Transactions on Learning Technologies (2017), 1-12.

[10] Sandy Claes and Andrew Vande Moere. 2015. The role of tangible interaction in exploring information on public visualization displays. In Proc. 4th Int. Symposium on Pervasive Displays. ACM, 201-207.

[11] Dan Cosley, Shyong K Lam, Istvan Albert, Joseph A Konstan, and John Riedl. 2003. Is seeing believing?: how recommender system interfaces affect users' opinions. In Proc. SIGCHI Conf. on Human factors in computing systems. ACM, 585-592.

[12] Ivania Donoso-Guzmán and Denis Parra. 2018. An Interactive Relevance Feedback Interface for Evidence-Based Health Care. In 23rd Int. Conf. on Intelligent User Interfaces (IUI '18). ACM, New York, NY, USA, 103-114. https://doi.org/10.1145/ 3172944.3172953

[13] Brynjar Gretarsson, John O'Donovan, Svetlin Bostandjiev, Christopher Hall, and Tobias Höllerer. 2010. Smallworlds: visualizing social recommendations. In Computer Graphics Forum, Vol. 29. Wiley Online Library, 833-842.

[14] Shiqiang Guo, Folami Alamudun, and Tracy Hammond. 2016. RÃl'suMatcher: A personalized rÃl'sumÃl-job matching system. Expert Systems with Applications 60 (2016), 169 - 182. https://doi.org/10.1016/j.eswa.2016.04.013

[15] F Maxwell Harper, Funing Xu, Harmanpreet Kaur, Kyle Condiff, Shuo Chang, and Loren Terveen. 2015. Putting users in control of their recommendations. In Proc. 9th ACM Conf. on Recommender Systems. ACM, 3-10.

[16] Chen He, Denis Parra, and Katrien Verbert. 2016. Interactive recommender systems: A survey of the state of the art and future research challenges and opportunities. Expert Systems with Applications 56, Supplement C (2016), 9 - 27. https://doi.org/10.1016/j.eswa.2016.02.013

[17] Islam A. Heggo and Nashwa Abdelbaki. 2018. Hybrid Information Filtering Engine for Personalized Job Recommender System. In The Int. Conf. on Advanced Machine Learning Technologies and Applications (AMLTA2018), Aboul Ella Hassanien, Mohamed F. Tolba, Mohamed Elhoseny, and Mohamed Mostafa (Eds.). Springer Int. Publishing, Cham, 553-563.

[18] Jonathan L Herlocker, Joseph A Konstan, and John Riedl. 2000. Explaining collaborative filtering recommendations. In Proc. CSCW' 00 . ACM, 241-250.

[19] Uta Hinrichs, Holly Schmidt, and Sheelagh Carpendale. 2008. EMDialog: Bringing information visualization into the museum. IEEE transactions on visualization and computer graphics 14, 6 (2008), 1181-1188.

[20] Rong Hu and Pearl Pu. 2011. Helping Users Perceive Recommendation Diversity.. In DiveRS@RecSys.43-50.

[21] Yucheng Jin, Bruno Cardoso, and Katrien Verbert. 2017. How do different levels of user control affect cognitive load and acceptance of recommendations?. In Proc. 4th foint Workshop on Interfaces and Human Decision Making for Recommender Systems co-located with ACM Conf. on Recommender Systems (RecSys 2017). CEURWS, 35-42.

[22] Yucheng Jin, Nava Tintarev, and Katrien Verbert. 2018. Effects of Personal Characteristics on Music Recommender Systems with Different Levels of Controllability. In Proceedings of the 12th ACM Conference on Recommender Systems (RecSys '18). ACM, New York, NY, USA, 13-21. https://doi.org/10.1145/3240323.3240358

[23] Todd Kulesza, Margaret Burnett, Weng-Keen Wong, and Simone Stumpf. 2015. Principles of explanatory debugging to personalize interactive machine learning. In Proc. 20th Int. Conf.Int. Intelligent User Interfaces. ACM, 126-137.

[24] Danielle H Lee and Peter Brusilovsky. 2009. Reinforcing recommendation using implicit negative feedback. In Int. Conf. User Modeling, Adaptation, and Personalization. Springer, 422-427.

[25] Martijn Millecamp, Nyi Nyi Htun, Yucheng Jin, and Katrien Verbert. 2018. Controlling Spotify recommendations: effects of personal characteristics on music recommender user Interfaces. In Proceedings of the 26th Conference on User Modeling, Adaptation and Personalization. ACM, 101-109.

[26] Till Nagel, Martina Maitan, Erik Duval, Andrew Vande Moere, Joris Klerkx, Kristian Kloeckl, and Carlo Ratti. 2014. Touching transport-a case study on visualizing metropolitan public transit on interactive tabletops. In Proc. 2014 Int. Working Conf. Advanced Visual Interfaces. ACM, 281-288.

[27] Suvi Nenonen, Heidi Rasila, Juha-Matti Junnonen, and Sam Kärnä. 2008. Customer Journey-a method to investigate user experience. In Proc. Euro FM Conf. 
Manchester. 54-63.

[28] John O’Donovan, Barry Smyth, Brynjar Gretarsson, Svetlin Bostandjiev, and Tobias Höllerer. 2008. PeerChooser: visual interactive recommendation. In Proc CHI '08. ACM, 1085-1088.

[29] Denis Parra and Peter Brusilovsky. 2015. User-controllable personalization: A case study with SetFusion. Int. Journal of Human-Computer Studies 78 (2015), 43 - 67. https://doi.org/10.1016/j.ijhcs.2015.01.007

[30] Denis Parra and Peter Brusilovsky. 2015. User-controllable personalization: A case study with SetFusion. International fournal of Human-Computer Studies 78 (2015), 43-67.

[31] Michael Reusens, Wilfried Lemahieu, Bart Baesens, and Luc Sels. 2017. A note on explicit versus implicit information for job recommendation. Decision Support Systems 98 (2017), $26-35$.

[32] Michael Reusens, Wilfried Lemahieu, Bart Baesens, and Luc Sels. 2018. Evaluating recommendation and search in the labor market. Knowledge-Based Systems 152 (2018), $62-69$

[33] Ian T. Ruginski, Alexander P. Boone, Lace M. Padilla, Le Liu, Nahal Heydari, Heidi S. Kramer, Mary Hegarty, William B. Thompson, Donald H. House, and Sarah H. Creem-Regehr. 2016. Non-expert interpretations of hurricane forecast uncertainty visualizations. Spatial Cognition \& Computation 16, 2 (2016), 154-172. https://doi.org/10.1080/13875868.2015.1137577

[34] James Schaffer, Tobias Höllerer, and John O’Donovan. 2015. Hypothetical Rec ommendation: A Study of Interactive Profile Manipulation Behavior for Recommender Systems.. In FLAIRS Conference. 507-512.

[35] Walid Shalaby, BahaaEddin AlAila, Mohammed Korayem, Layla Pournajaf, Khalifeh AlJadda, Shannon Quinn, and Wlodek Zadrozny. 2017. Help me find a job: A graph-based approach for job recommendation at scale. In Big Data (Big Data), 2017 IEEE Int. Conf. on. IEEE, 1544-1553.

[36] B. Shneiderman. 1996. The eyes have it: a task by data type taxonomy for information visualizations. In IEEE Symposium Visual Languages. IEEE, 336-343. https://doi.org/10.1109/VL.1996.545307

[37] Zheng Siting, Hong Wenxing, Zhang Ning, and Yang Fan. 2012. Job recommender systems: a survey. In Computer Science \& Education (ICCSE), 2012 7th Int. Conf. IEEE, 920-924.

[38] Anthony Tang, Melanie Tory, Barry Po, Petra Neumann, and Sheelagh Carpendale 2006. Collaborative coupling over tabletop displays. In Proc. SIGCHI Conf. Human Factors in computing systems. ACM, 1181-1190.

[39] Alice Thudt, Uta Hinrichs, and Sheelagh Carpendale. 2012. The bohemian book shelf: supporting serendipitous book discoveries through information visualization. In Proc. SIGCHI Conf.Int. Human Factors in Computing Systems. ACM, 1461-1470.

[40] Chun-Hua Tsai and Peter Brusilovsky. 2017. Enhancing Recommendation Diversity Through a Dual Recommendation Interface. In 4 th foint Workshop on Interfaces and Human Decision Making for Recommender Systems (IntRS) 2017. 10.

[41] Katrien Verbert, Denis Parra, Peter Brusilovsky, and Erik Duval. 2013. Visualizing recommendations to support exploration, transparency and controllability. In Proc. 2013 Int. Conf. on Intelligent user interfaces. ACM, 351-362. 